\title{
Distinction between benign and malignant soft tissue tumors based on an ultrasonographic evaluation of vascularity and elasticity
}

\author{
SHUSA OHSHIKA, TATSURO SARUGA, TETSUYA OGAWA, HIROYA ONO and YASUYUKI ISHIBASHI
}

Department of Orthopaedic Surgery, Hirosaki University Graduate School of Medicine, Hirosaki, Aomori 036-8562, Japan

Received May 1, 2020; Accepted December 30, 2020

DOI: 10.3892/ol.2021.12542

\begin{abstract}
The initial diagnostic distinction between benign and malignant soft tissue tumors is critical for decisions regarding the appropriate course of treatment. The current study aimed to evaluate the vascularity and elasticity of soft tissue tumors by superb microvascular imaging and shear wave elastography using ultrasonography (US), to determine their usefulness in distinguishing malignant soft tissue tumors, and to further establish the diagnostic accuracy and usefulness of a scoring system (SS) based on these evaluations. The present study used 167 lesions of soft tissue tumors examined by US prior to biopsy, surgery and pathological tissue diagnosis. The vascularity index (VI) and the maximal shear velocity (MSV), as indices of vascularity and elasticity respectively, were evaluated using US. The tumor size and depth were also evaluated via magnetic resonance imaging (MRI). Based on the odds ratio of these parameters determined by multivariate logistic regression analysis, an original SS was established to identify the malignancy of soft tissue tumors. VI and MSV exhibited significantly high values for malignant tumors. Tumor size was also significantly larger for malignant than benign tumors. The areas under the curves (AUCs) of the receiver operating characteristic analysis for VI, MSV and tumor size were 0.75 , 0.84 and 0.69 , respectively, indicating that these methods were effective for the diagnosis of malignancy. An original SS consisting of VI, MSV and tumor size, excluding tumor depth, was established, and revealed an AUC value of 0.90, with $93.6 \%$ sensitivity and $79.2 \%$ specificity for malignancy distinction. US evaluation of vascularity and elasticity was
\end{abstract}

Correspondence to: Dr Shusa Ohshika, Department of Orthopaedic Surgery, Hirosaki University Graduate School of Medicine, 5 Zaifu-cho, Hirosaki, Aomori 036-8562, Japan

E-mail: ohshika@hirosaki-u.ac.jp

Abbreviations: AUC, area under the curve; MRI, magnetic resonance imaging; MSV, maximal shear velocity; ROI, region of interest; ROC, receiver operating characteristic; SE, strain elastography; SMI, superb microvascular imaging; SS, scoring system; SWE, shear wave elastography; US, ultrasonography; VI, vascularity index

Key words: soft tissue tumor, ultrasonography, vascularity, elasticity, scoring system an effective technique to distinguish malignant soft tissue tumors, and the current SS based on US evaluations including tumor size via MRI demonstrated a high diagnostic accuracy for malignant soft tissue tumors.

\section{Introduction}

Soft tissue tumors in the extremities and the trunk can exhibit a variety of pathological features, making them potentially difficult to diagnose (1). Although magnetic resonance imaging (MRI) results are typically used for diagnosis, the examination frequently lacks specific findings. Even radiological diagnosticians and medical specialists of bone/soft tissue tumors often struggle to distinguish between benign and malignant tumors based on such imaging data (2). Since inappropriate primary care adversely affects patients' prognoses, a clear distinction between benign and malignant tumors upon the initial diagnosis is essential $(3,4)$.

Ultrasonography (US) is a simple imaging diagnostic tool with low invasiveness that has improved through technical advancements and is increasingly being utilized in orthopedic cases (5). Vascularity evaluation using color Doppler and power Doppler is also useful in distinguishing between benign and malignant tumors when diagnosing soft tissue tumors (6-8). The Giovagnorio classification (6) divides the vascular distribution into 4 types and has been used in various studies (8-10). Furthermore, a scoring system (SS) evaluating vascularity, tumor size, echogenicity, and internal structure findings can improve the accuracy of benign and malignant tumor distinction $(9,10)$. Strain elastography $(\mathrm{SE})$ is a method for elasticity evaluation based on the strain on the tissue upon the application of pressure via probes. Using SE to evaluate the elasticity can also be useful in the diagnosis of soft tissue tumors (11-16).

Among the newly improved evaluation techniques for US, superb microvascular imaging (SMI) facilitates the assessment of lower flow vascularity better than color Doppler and power Doppler $(17,18)$. In addition, shear wave elastography (SWE) is useful to evaluate tissue elasticity by calculating the velocity of the shear waves passing through the tissue (19-22). Unlike SE, SWE is a simple method because it does not require the application of pressure via probes and provides quantitative data on the stiffness of the tissue. These techniques have been applied to other anatomical regions such as the thyroid gland, mammary gland, liver, and prostate gland (17-22). 
However, the usefulness of SMI and SWE in the diagnosis of soft tissue tumors remains unclear.

This study aimed to determine: i) the usefulness of vascularity and elasticity evaluation, using SMI and SWE, respectively, to distinguish between benign and malignant soft tissue tumors, and ii) the diagnostic accuracy of soft tissue tumors by establishing an original SS based on vascularity and elasticity assessments.

\section{Materials and methods}

Patient characteristics. This retrospective study was approved by the Human Research Ethics Committee of the Hirosaki University Graduate School of Medicine (Aomori, Japan; reference no. 2019-1023), and the requirement for informed consent was waived because of the anonymous nature of the data. This study targeted consecutive cases that were examined by US prior to biopsy, surgery, and pathological tissue diagnosis at our hospital from April 2016 to September 2018. Medical records, US data, and MRI data were investigated in April 2019, and the cases without sufficient data were excluded. A total of 167 lesions in 164 cases (86 male, 78 female) was enrolled in the present study. The mean age was 56 years (range: 5 to 92). Pediatric cases were only eight (5 to 16 years). There were 47 targeted lesions in the upper extremities, 76 in the lower extremities, and 44 in the trunk. The pathological tissue diagnosis was based on the World Health Organization classification 2013 (1), and was divided into 3 groups: benign, intermediate, and malignant (Table I). Non-tumoral benign lesion types, such as ganglion, atheroma, and hematoma were also targeted. One case of an intramuscular glomus tumor, which was a non-typical case that was diagnosed with uncertain malignant potential, was classified into the intermediate group in this study.

Evaluation of image findings on US and MRI. The 4 items evaluated were as follows: vascularity index (VI) for SMI, maximal shear velocity (MSV) for SWE, and tumor size and tumor depth on MRI. All US examinations and MRI reviews were conducted by a single musculoskeletal oncologist with 19 years of experience. The Aplio 500 (Toshiba Medical Systems Corporation) was used as the US diagnostic device and a linear-type probe PLT-1005BT was used for the evaluations. The vascularity of the tumors was evaluated by SMI (Fig. 1A). The vascular ratio in the region of interest (ROI) was quantified. The mean vascularity ratio at three measured positions was defined as the VI. The elasticity of the tumors was evaluated by SWE in the same intratumoral areas that were measured by SMI. Small circular ROIs with a diameter of $1 \mathrm{~mm}$ were placed in the rectangular ROI of SWE to calculate shear velocity (Fig. 1B). The maximal value among shear velocity values was defined as the MSV. The size of the ROI was equal to the tumor size when the entire tumor fit within the width of the probe. When the tumor was larger than the width of the probe, the ROI was adjusted to evaluate the tumor from the shallow to central parts. The maximal length of the tumor visible via MRI was used as the tumor size for all cases because most tumors were large and unmeasurable by the US device. The depth of the tumor was also evaluated by MRI for all cases. Tumors were divided into superficial or deep categories based on their location relative to the fascia.
Statistical analyses. The tumor size values were normally distributed, those of VI and MSV were not normally distributed by the Shapiro-Wilk test. Values of tumor size, VI, and MSV were presented as medians with interquartile range, and medians among the 3 groups (benign, intermediate, and malignant) were compared by the Kruskal-Wallis test followed by Dunn's test as a post hoc analysis. The depth (deep/superficial) was compared using a Fisher's exact test. To investigate the cut-off values of the tumor size, VI, and MSV, the receiver operating characteristic (ROC) analysis was performed. In this analysis, the area under the curve (AUC), odds ratio, and the respective cut-off values based on the malignancy were calculated. Using the cut-off values of the tumor size (large/small), VI (hyper/hypo vascularity) and MSV (hard/soft) from the ROC analysis, multivariate logistic regression analysis was performed, with the malignancy against the benign or intermediate tumors as a dependent variable, and the presence of tumor size, VI, MSV, and depth. Data input and data analysis were performed using SPSS version 25.0J (SPSS Inc.), and the Excel statistical software BellCurve for Excel (Version 3.0; Social Survey Research Information Co., Ltd.). A P-value <0.05 was considered statistically significant.

Establishment and evaluation of an original SS. Based on the odds ratios of the multivariate logistic regression analysis, an original SS was established. A cut-off value of the SS score for malignancy was also determined by a ROC analysis, and the sensitivity and specificity were calculated. The median scores of the SS between the three groups were compared using Kruskal-Wallis test followed by Dunn's test as a post hoc analysis. In addition, the scores for each soft tissue tumor, in which the number of lesions was 3 or more, were evaluated.

\section{Results}

Evaluation of image findings on US and MRI. Values of VI and MSV were significantly higher in the malignant group than in the benign and intermediate group $(\mathrm{P}<0.001$, respectively) (Table II). There were no significant differences in the values of VI between the benign and intermediate groups $(\mathrm{P}=0.592)$. While the tumor size in the intermediate and malignant groups was significantly larger than that of the benign group $(\mathrm{P}<0.001)$, there was no significant difference in tumor size between the intermediate and malignant groups $(\mathrm{P}=0.109)$. Ratio of deep lesion was higher in the intermediate and malignant groups $(\mathrm{P}=0.005)$.

ROC analysis (Fig. 2) indicated the cut-off value of tumor size for malignancy was $7 \mathrm{~cm}$ (AUC: 0.69, $\mathrm{P}<0.001$, odds: $4.0,95 \%$ CI: $0.61-0.78)$. The cut-off value of VI was 5.3\% (AUC: $0.75, \mathrm{P}<0.001$, odds: 7.0, 95\% CI: 0.68-0.83). The cut-off value of MSV was $7.9 \mathrm{~m} / \mathrm{sec}$ (AUC: $0.84, \mathrm{P}<0.001$, odds: $18.5,95 \%$ CI: 0.77-0.91).

Establishment and evaluation of original SS. All the 4 items (VI, MSV, tumor size and tumor depth) were entered into the multivariate logistic regression analysis, and the three values (VI, MSV and tumor size) were selected as independent risk factors (Table III). The odds ratios for malignancy against other groups were 8.74, 18.81 and 6.03 for VI, MSV and tumor size, respectively. An original SS consisting of these 
A

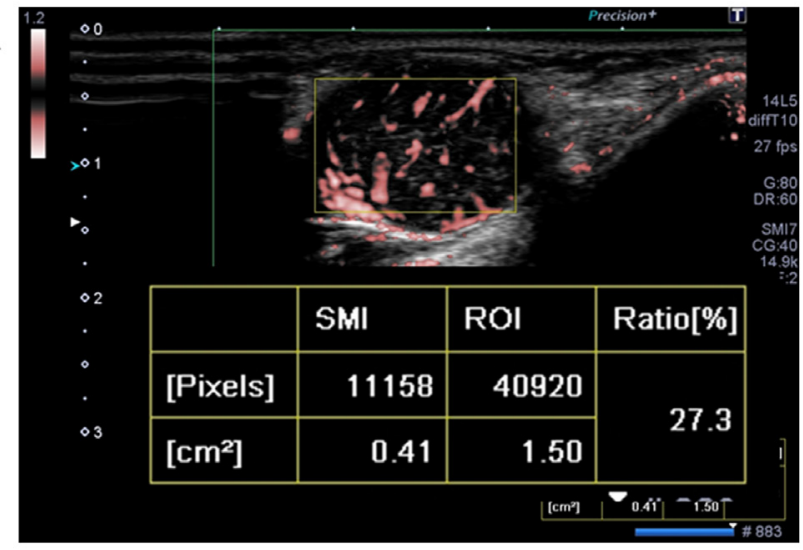

B

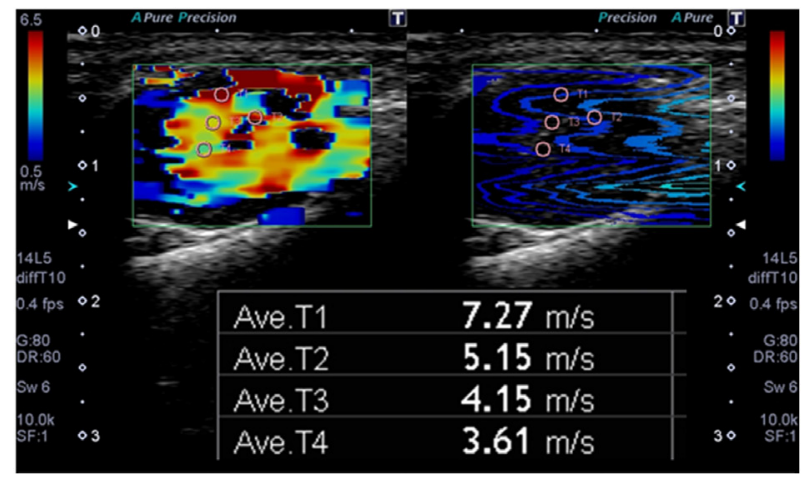

Figure 1. Ultrasonographic imaging of a brachial schwannoma in a 29-year-old female. (A) Vascularity evaluation by superb microvascular imaging. Pink indicates vascularity. The ROI was adjusted to the size of tumors, and revealed a vascularity ratio of $27.3 \%$. The mean ratio at three measured positions was defined as the vascularity index. (B) Elasticity evaluation by SWE. The colors indicate tumor elasticity; hard tumors with high shear velocity are red and soft tumors with low shear velocity are blue. Small circular ROIs with a diameter of $1 \mathrm{~mm}$ were placed in the rectangular ROI of SWE to calculate shear velocity. Measured shear velocity values are presented in the lower screen. The maximal value among shear velocity values calculated within the rectangular ROI was defined as the maximal shear velocity, which was $7.27 \mathrm{~m} / \mathrm{sec}$. ROI, region of interest; SMI, superb microvascular imaging; SWE, shear wave elastography; Ave, average.

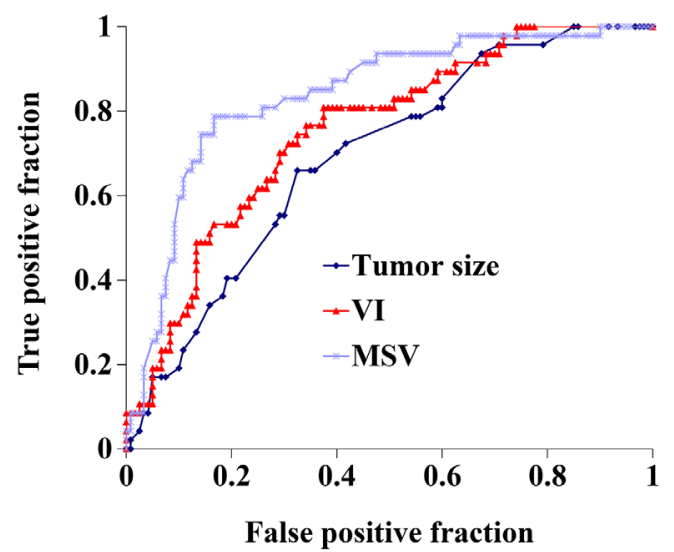

Figure 2. ROC analysis for tumor size, VI and MSV. The area under the curve values for tumor size, VI and MSV were $0.69,0.75$ and 0.84 , respectively. The cut-off values with respect to malignancy for tumor size, VI and MSV were $7 \mathrm{~cm}, 5.3 \%$ and $7.9 \mathrm{~m} / \mathrm{sec}$, respectively. ROC, receiver operating characteristic; VI, vascularity index; MSV, maximal shear velocity.

3 items (VI, MSV and tumor size) was established based on the odds ratio for identifying the malignancy of the soft tissue
Table I. Number of patients diagnosed with different forms of pathological tumor.

\begin{tabular}{cc}
\hline Tumor type & $\begin{array}{c}\text { Number of } \\
\text { patients }\end{array}$
\end{tabular}

Benign soft tissue tumors $(n=99)$

Lipoma

Schwannoma

Vascular malformation

Epidermal cyst

Spindle cell lipoma

Tenosynovial giant cell tumor (localized type)

Intramuscular myxoma

Chronic expanding hematoma

Fibroma of tendon sheath

Ganglion

Elastofibroma

Hematoma

Desmoplastic fibroblastoma

Neurofibroma

Hibernoma

Angiolipoma

Nodular fasciitis

Intermediate soft tissue tumors $(n=21)$

Atypical lipomatous tumor

Desmoid type fibromatosis

Plantar fibromatosis

Dermatofibrosarcoma protuberans

Soft tissue recurrence of bone GCTs

Glomus tumor (uncertain malignant potential)

Malignant soft tissue tumors $(n=47)$

Myxofibrosarcoma

Myxoid liposarcoma

Undifferentiated pleomorphic sarcoma

Soft tissue metastasis of carcinoma

Rhabdomyosarcoma

Leiomyosarcoma

Extraskeletal myxoid chondrosarcoma

Extraskeletal osteosarcoma

Synovial sarcoma

Dedifferentiated liposarcoma

High grade sarcoma

Malignant lymphoma

Low-grade fibromyxoid sarcoma

Clear cell sarcoma tumors (Table IV). The score of the 3 items summed for each lesion ranged from 0 to 5.5 points.

ROC analysis (Fig. 3) showed that the cut-off value of score of original SS for malignancy was estimated as 2.5 points (AUC: 0.90, P<0.001, odds: 55.7, 95\% CI: 0.85-0.96). Based on this cut-off value, original SS showed $93.6 \%$ sensitivity and $79.2 \%$ specificity to identify malignancy. In the score distribution by using the original SS, as the score increased, 


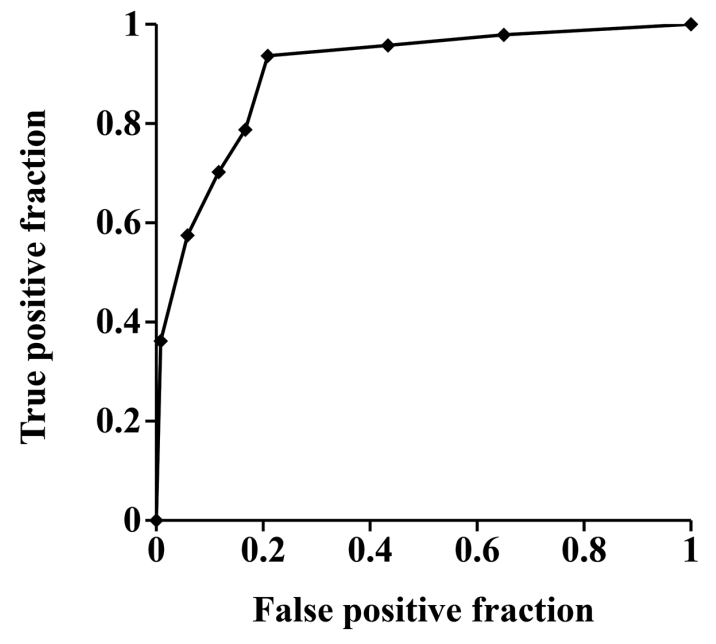

Figure 3. ROC analysis for the newly established original SS. The area under the curve value for the total score of the original SS was 0.90, and the cut-off value of the original SS for malignancy was estimated at 2.5 points. The original SS demonstrated $93.6 \%$ sensitivity and $79.2 \%$ specificity. ROC, receiver operating characteristic; SS, scoring system.

the incidence of malignant lesions increased (Fig. 4A). Three (1 myxofibrosarcoma, 1 soft tissue metastasis of carcinoma, 1 malignant lymphoma) out of the 47 malignant lesions had scores $<2.5$ points and the other 44 had scores $\geq 2.5$ points. Nineteen of the 99 benign lesions and 6 of the 21 intermediate lesions had scores $\geq 2.5$ points. The median and interquartile range of scores for the benign, intermediate, and malignant groups were $1.0(0-1.5)$ (95\% CI: 0.94-1.47), 1.5 (1.0-2.5) (95\% CI: $1.12-2.55)$, and 4.5 (3.0-5.5) (95\% CI: 3.73-4.55), respectively. The original SS score of the malignant group was significantly higher than that of the other groups $(\mathrm{P}<0.001)$ (Fig. 4B). There was no significant difference between the benign and the intermediate group $(\mathrm{P}=0.350)$. Representative scores of soft tissue tumors were also calculated (Table SI). In the benign group, schwannoma, vascular malformation, and spindle cell lipoma showed relatively high scores compared to the other benign lesions. In the intermediate group, desmoid type fibromatosis showed a high score compared to the other lesions. There were no significant differences between the three lipomatous tumors, namely, lipoma, spindle cell lipoma, and atypical lipomatous tumor. In the malignant group, all lesions showed a high score, and there was no significant difference between them.

\section{Discussion}

In this study, our results revealed significantly high VI and MSV values for malignant group and demonstrated the usefulness of these techniques in distinguishing between benign or intermediate and malignant group. Furthermore, the original SS based on vascularity and elasticity assessments by US in conjunction with tumor size on MRI offered high diagnostic accuracy for malignant group. This study is, to the best of our knowledge, the first report to demonstrate the usefulness of both vascularity and elasticity to distinguish between benign or intermediate and malignant soft tissue tumors.

Evaluation of tumor vascularity using SMI established the technique's efficacy in distinguishing between benign or
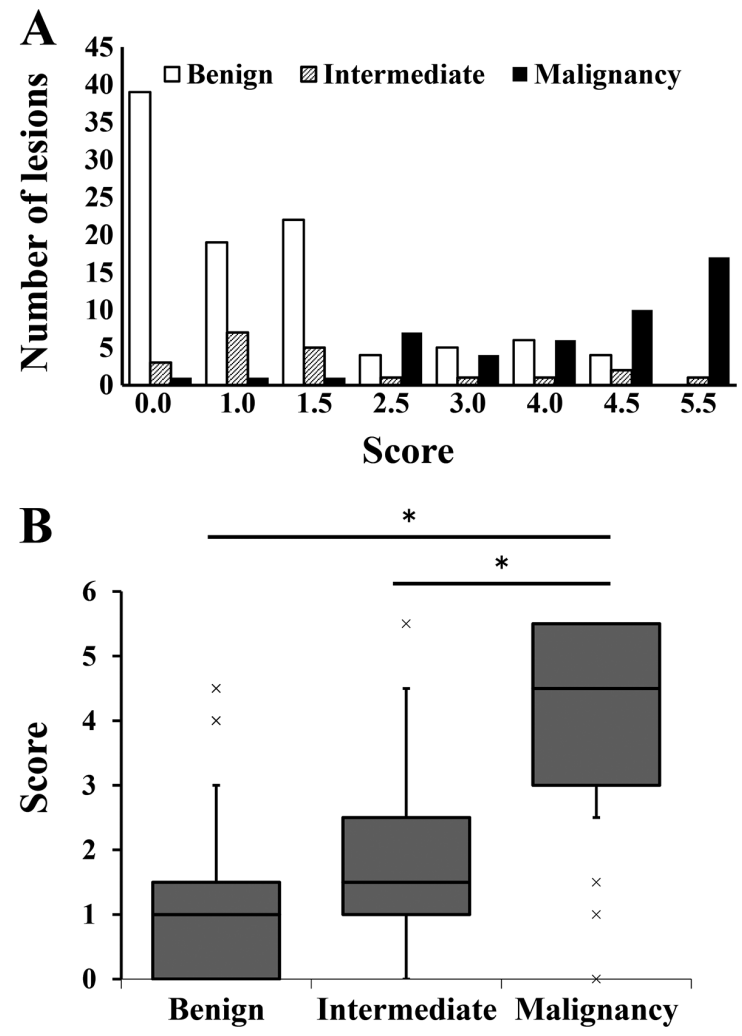

Figure 4. Score distribution of the each case and the mean score of the respective groups evaluated using the original scoring system. (A) Score distribution of each case. As the score increased, the incidence of malignant lesions increased. (B) The median scores for the benign, intermediate and malignant groups were $1.0,1.5$ and 4.5 , respectively. In the box plot, the values are presented as medians, and the box indicates the interquartile range of the scores. The bar indicates the $90 \%$ range, and $\mathrm{x}$ indicates the range outside $90 \%$. These parameters were compared using the Kruskal-Wallis test followed by Dunn's test as a post hoc analysis. " $\mathrm{P}<0.05$.

intermediate, and malignant soft tissue tumors. Malignant solid tumors show rich vascularity since tumor angiogenesis is enhanced in invasive and progressive malignant solid tumors (23). Many examiners frequently assess the distribution of intratumoral vascularity using the Giovagnorio classification for evaluation in US (6,8-10). Oebisu et al reported color Doppler US with a contrast medium increased the diagnostic accuracy of malignant soft tissue tumors based on the Giovagnorio classification, since the use of the contrast medium made evaluation of the intratumoral vascularity possible for a longer duration and in more detail (8). We attempted the quantification of the intratumoral vascularity by SMI instead of the vascular distribution by color Doppler, since VI values were significantly higher for the malignant group than benign or intermediate group. This study is, to the best of our knowledge, the first report to demonstrate the usefulness of quantification of the intratumoral vascularity by SMI to distinguish between benign or intermediate and malignant soft tissue tumors. SMI is a non-invasive and easy-to-use technique to enable the visualization of low flow and fine vascularity without contrast medium and could be used for initial diagnostic distinction of soft tissue tumors.

SWE for the evaluation of tumor elasticity was also useful for making a distinction between benign or intermediate, and malignant soft tissue tumors. Malignant lesions in other organs, 
Table II. Comparison of VI, MSV, tumor size and tumor depth between each group.

\begin{tabular}{|c|c|c|c|}
\hline Method & Benign & Intermediate & Malignancy \\
\hline \multicolumn{4}{|l|}{ Vascularity index (\%) } \\
\hline Median & 2.4 & 3.1 & $10.0^{\mathrm{a}, \mathrm{b}}$ \\
\hline Interquartile range & $(0.2-6.0)$ & $(1.3-7.6)$ & $(5.0-12.0)$ \\
\hline \multicolumn{4}{|c|}{ Maximal shear velocity $(\mathrm{m} / \mathrm{sec})$} \\
\hline Median & 6.1 & 7 & $8.3^{\mathrm{a}, \mathrm{b}}$ \\
\hline Interquartile range & $(3.4-7.7)$ & $(5.9-7.6)$ & $(7.9-8.7)$ \\
\hline \multicolumn{4}{|l|}{ Tumor size (cm) } \\
\hline Median & 5.0 & 7.8 & $8.0^{\mathrm{a}}$ \\
\hline Interquartile range & $(3.0-7.4)$ & $(3.8-14.6)$ & $(5.0-12.0)$ \\
\hline \multicolumn{4}{|l|}{ Depth } \\
\hline Superficial lesion (n) & 37 & 2 & 8 \\
\hline Deep lesion (n) & 62 & 19 & 39 \\
\hline Ratio of deep lesion (\%) & 62.6 & 90.5 & $83.0^{\mathrm{c}}$ \\
\hline
\end{tabular}

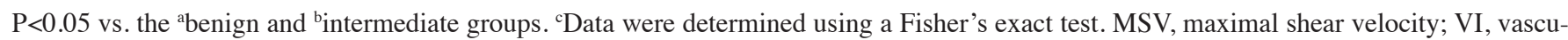
larity index.

Table III. Multivariate logistic regression analysis of each method.

\begin{tabular}{|c|c|c|c|c|c|c|c|c|}
\hline \multirow[b]{2}{*}{ Method } & \multicolumn{4}{|c|}{ Crude } & \multicolumn{4}{|c|}{ Adjusted } \\
\hline & $\mathrm{B}$ & P-value & Odds ratio & $95 \% \mathrm{CI}$ & $\mathrm{B}$ & P-value & Odds ratio & $95 \% \mathrm{CI}$ \\
\hline Vascularity index & 1.80 & $<0.001$ & 6.06 & $2.8-12.9$ & 2.17 & $<0.001$ & 8.74 & $3.0-25.2$ \\
\hline Maximal shear velocity & 2.92 & $<0.001$ & 18.5 & $7.9-43.2$ & 2.9 & $<0.001$ & 18.81 & $6.9-51.2$ \\
\hline Tumor size & 1.39 & $<0.001$ & 4.02 & $2.0-8.2$ & 1.80 & 0.001 & 6.03 & $2.2-16.7$ \\
\hline Depth & 0.85 & 0.049 & 2.35 & $1.0-5.5$ & -0.01 & 0.983 & 0.99 & $0.31-3.2$ \\
\hline
\end{tabular}

B, partial regression coefficient; CI, confidence interval.

Table IV. An original scoring system based on the odds ratio from the multivariate logistic regression analysis for the identification of soft tissue malignancy.

\begin{tabular}{lc}
\hline Method & Points \\
\hline Tumor size $(\mathrm{cm})$ & \\
$<7$ & 0.0 \\
$\geq 7$ & 1.0 \\
Vascularity index $(\%)$ & \\
$<5.3$ & 0.0 \\
$\geq 5.3$ & 1.5 \\
Maximal shear velocity $(\mathrm{m} / \mathrm{sec})$ & \\
$<7.9$ & 0.0 \\
$\geq 7.9$ & 3.0 \\
Total & $0.0-5.5$ \\
\hline
\end{tabular}

such as the breast, prostate, and thyroid, showed high shear wave velocity indicating stiffness on palpation (24-26). Recent studies reported that SWE was not useful for making a distinction between benign and malignant tumors and malignant musculoskeletal lesions trended toward a lower shear wave velocity compared to benign lesions (26-29), indicating that malignant lesions are softer than benign lesions. In our study, MSV values in SWE were significantly higher for the malignant group than the benign group, signifying malignant lesions are stiffer, which is in accordance with previous SE studies that malignant soft tissue lesions tended to be stiffer compared to the benign lesions $(13,15,16)$. The different results in SWE between previous studies (27-30) and ours could be explained by the differences of implemented US devices and evaluation methods. Previous studies used US devices made in Euro-American companies such as Siemens Acuson and LOGIQ-E9, and evaluated each in different ways such as mean of multi-velocity readings and use of fixed sized SWE rectangular box. We used the Aplio 500 made in Japan, and assessed the maximal values among the shear velocity values calculated in our study. Other reasons may be the differences in clinical characteristics of targeted lesions such as size, depth, and pathological heterogeneous nature.

Tumor size is a universal finding suggesting malignant soft tissue tumors (31). As malignant soft tissue tumors grow, 
they may become firmer because intratumoral necrotic tissue enlarges due to hypoxia or intratumoral pressure is increased due to compression between surrounding tissues such as muscle and fascia. Our study had an increased number of larger malignant soft tissue tumors that were unmeasurable by the US device compared to previous SWE reports (28-30). Our SWE results are similar to the common clinical presentation of most soft tissue sarcomas, which tend to be found on palpation with elastic to hard firmness. The depth of the tumor is also an important finding suggesting malignant soft tissue tumors. The depth of the malignant group was significantly deeper compared to that of the benign group in univariate analysis in our study. However, we had not include the depth of the tumor in our SS while considering the results of multivariate logistic regression analysis. Indeed, notation considering the depth of the tumor was eliminated in the new TNM classification of soft tissue sarcoma (31).

Our SS based on vascularity and elasticity evaluated by US showed high diagnostic accuracy for malignant soft tissue tumors. Nagano et al used an SS consisting of 4 items (i.e., tumor size, echogenicity, internal texture, and vascular distribution with color Doppler) to report sarcoma diagnosis capability with an AUC value of $0.88,85.1 \%$ sensitivity, and $86.9 \%$ specificity (9). Recently, Morii et al used an SS consisting of tumor size, vascular distribution with power Doppler, and tumor margins to report its usefulness in sarcoma diagnoses with an AUC value of $0.85,82.5 \%$ sensitivity, and $73.2 \%$ specificity (10). Our SS showed higher AUC values and sensitivity compared to previous reports, and also showed the possibility to distinguish soft tissue tumors with same differentiation such as lipomas, spindle cell lipomas, and atypical lipomatous tumors. In our hospital, most of the patients were referred to our department with MRI data without contrast enhancement, and we had evaluated the intratumoral vascularity and elasticity by SMI and SWE at the date of first visit. Furthermore, we performed needle biopsy under US guidance for an early diagnosis if soft tissue lesions of the referral cases had high vascularity and elasticity. Evaluation of tumor size via MRI is an uncomplicated approach for both non-medical and medical specialists. US examination is also safe, low-cost, and easy to use for a non-medical specialist. Our SS based on US evaluation including tumor size via MRI may be universally suitable for initial and early diagnostic distinction.

This study, while emphasizing the importance of elasticity and vascularity, has pitfalls of US such as the potential for false negatives and false positives. Several mucinous malignant tumors (4 myxofibrosarcomas, 2 myxoid liposarcomas, and 1 low grade fibromyxoid sarcoma) showed low MSV $(<7.9 \mathrm{~m} / \mathrm{sec}$ ) values as false negatives in our study. Two myxofibrosarcomas and 1 myxoid liposarcoma showed considerably low MSV (4.4, 2.2 and $4.9 \mathrm{~m} / \mathrm{sec})$. Evaluating the vascularity also enhances the likelihood of acquiring false negatives for large malignant tumors with wide range necrosis, which exhibit low values for VI. Meanwhile, schwannomas and desmoid-type fibromatoses exhibited high values for vascularity and elasticity and were frequently determined as false positives. US provides information using a non-invasive technique at a low cost and is an essential imaging examination tool for the initial diagnosis of soft tissue tumors despite these pitfalls.
Our study had several limitations. First, all examinations, and evaluations were performed by the first author, a medical specialist for bone/soft tissue tumors. The retrospective nature of the study is also a limitation. Poor inter-rater reliability may be caused by the differences of US technique between specialists and non-specialists, especially with regard to location of the probe and selection of ROI. Thus, in the future, prospective studies with examinations conducted by medical specialists and non-medical specialists should be conducted to determine the repeatability and reliability of the results. Additionally, patient population was heterogeneous with regard to tumor size, depth, and pathological diagnosis. It is favorable to compare between benign and malignant soft tissue tumors with the same sizes and depths, but malignant lesions are often larger and deeper than benign ones in clinical practice. Sample size was too small to compare between benign or intermediate, and malignant soft tissue tumors under the same conditions. A multicenter study with larger sample size is necessary to elucidate the US characteristics such as tumor size and depth under similar conditions. A multicenter study might also reveal novel US findings for specific types of soft tissue tumors such as mucinous and lipomatous soft tissue tumors. Other limitation of our study is the selection bias due to the indication of the surgery. We included only cases in which the pathological tissue diagnosis was proven. We should consider that the cut-off values and the SS setting might have changed if our study had included all benign and intermediate soft tissue tumors that were evaluated by US but not resected.

In conclusion, evaluating vascularity by SMI and elasticity by SWE is a useful technique to distinguish between benign or intermediate and malignant tumors, even if the evaluations are performed separately. Furthermore, our SS established based on these evaluations including tumor size via MRI showed high diagnostic accuracy for malignant soft tissue tumors. Therefore, an SS based on the US evaluation of vascularity and elasticity is a useful initial diagnostic tool for soft tissue tumors.

\section{Acknowledgements}

The authors would like to thank Dr Sasaki E (Department of Orthopaedic Surgery, Hirosaki University Graduate School of Medicine, Hirosaki, Japan) for his assistance in the statistical analyses of the data.

\section{Funding}

No funding was received.

\section{Availability of data and materials}

The datasets used and/or analyzed during the current study are available from the corresponding author on reasonable request.

\section{Authors' contributions}

SO, TS, TO, HO and YI conceived and designed the current study. SO, TS, TO and HO treated and observed patients at Hirosaki University. SO performed ultrasonography examinations and MRI reviews, prepared the manuscript and acquired 
the data. TS and TO confirmed the authenticity of all the raw data. YI supervised the current study. All authors participated in the interpretation of the results and have read and approved the final version of the manuscript.

\section{Ethics approval and consent to participate}

All procedures performed in studies involving human participants were in accordance with the ethical standards of the institutional and/or National Research Committee and with the 1964 Helsinki declaration and its later amendments or comparable ethical standards. The study protocol was approved by Human Research Ethics Committee of the Hirosaki University Graduate School of Medicine (Aomori, Japan; reference no. 2019-1023). The present study was performed using a retrospective design. The requirement for informed consent was waived because of the anonymous nature of the data. The opt-out approach was used.

\section{Patient consent for publication}

Not applicable.

\section{Competing interests}

The authors declare that they have no competing interests.

\section{References}

1. Fletcher CD, Bridge JA, Hogendoorn PC and Mertens F (eds) World Health Organization classification of tumours of soft tissue and bone. 4th edition. IARC Press, Lyon, 2013.

2. Moulton JS, Blebea JS, Dunco DM, Braley SE, Bisset GS III and Emery KH: MR imaging of soft-tissue masses: Diagnostic efficacy and value of distinguishing between benign and malignant lesions. AJR Am J Roentgenol 164: 1191-1199, 1995.

3. Arai E, Nishida Y, Tsukushi S, Wasa J and Ishiguro N: Clinical and treatment outcomes of planned and unplanned excisions of soft tissue sarcomas. Clin Orthop Relat Res 468: 3028-3034, 2010.

4. Pretell-Mazzini J, Barton MD Jr, Conway SA and Temple HT: Unplanned excision of soft-tissue sarcomas: Current concepts for management and prognosis. J Bone Joint Surg Am 97: 597-603 2015.

5. Blankstein A: Ultrasound in the diagnosis of clinical orthopedics: The orthopedic stethoscope. World J Orthop 2: 13-24, 2011.

6. Giovagnorio F, Andreoli C and De Cicco ML: Color Doppler sonography of focal lesions of the skin and subcutaneous tissue. J Ultrasound Med 18: 89-93, 1999.

7. Futani H, Yamagiwa T, Yasojimat H, Natsuaki M, Stugaard M and Maruo S: Distinction between well-differentiated liposarcoma and intramuscular lipoma by power Doppler ultrasonography. Anticancer Res 23: 1713-1718, 2003.

8. Oebisu N, Hoshi M, Ieguchi M, Takada J, Iwai T, Ohsawa M and Nakamura H: Contrast-enhanced color Doppler ultrasonography increases diagnostic accuracy for soft tissue tumors. Oncol Rep 32: 1654-1660, 2014

9. Nagano S, Yahiro Y, Yokouchi M, Setoguchi T, Ishidou Y, Sasaki H, Shimada H, Kawamura I and Komiya S: Doppler ultrasound for diagnosis of soft tissue sarcoma: Efficacy of ultrasound-based screening score. Radiol Oncol 49: 135-140, 2015.

10. Morii T, Kishino T, Shimamori N, Motohashi M, Ohnishi H, Honya K, Aoyagi T, Tajima T and Ichimura S: Differential diagnosis between benign and malignant soft tissue tumors utilizing ultrasound parameters. J Med Ultrason 2001 45: 113-119, 2018.

11. Lalitha P, Reddy MC and Reddy KJ: Musculoskeletal applications of elastography: A pictorial essay of our initial experience. Korean J Radiol 12: 365-375, 2011.
12. Lee YH, Song HT and Suh JS: Use of strain ratio in evaluating superficial soft tissue tumors on ultrasonic elastography. J Med Ultrason 2001 41: 319-323, 2014.

13. Magarelli N, Carducci C, Bucalo C, Filograna L, Rapisarda S, De Waure C, Dell'Atti C, Maccauro G, Leone A and Bonomo L: Sonoelastography for qualitative and quantitative evaluation of superficial soft tissue lesions: A feasibility study. Eur Radiol 24: 566-573, 2014.

14. Kim SJ, Park HJ and Lee SY: Usefulness of strain elastography of the musculoskeletal system. Ultrasonography 35: 104-109, 2016.

15. Park HJ, Lee SY, Lee SM, Kim WT, Lee S and Ahn KS: Strain elastography features of epidermoid tumours in superficial soft tissue: Differences from other benign soft-tissue tumours and malignant tumours. Br J Radiol 88: 20140797, 2015.

16. Hahn S, Lee YH, Lee SH and Suh JS: Value of the strain ration on ultrasonic elastography for differentiation of benign and malignant soft tissue tumors. J Ultrasound Med 36: 121-127, 2017.

17. Machado P, Segal S, Lyshchik A and Forsberg F: A novel microvascular flow technique: Initial results in thyroids. Ultrasound Q 32: 67-74, 2016.

18. Ohno Y, Fujimoto T and Shibata Y: A new era in diagnostic ultrasound, superb microvascular imaging: Preliminary results in pediatric hepato-gastrointestinal disorders. Eur J Pediatr Surg 27: 20-25, 2017.

19. Lin P, Chen M, Liu B, Wang S and Li X: Diagnostic performance of shear wave elastography in the identification of malignant thyroid nodules: A meta-analysis. Eur Radiol 24: 2729-2738, 2014.

20. Liu B, Zheng Y, Huang G, Lin M, Shan Q, Lu Y, Tian W and Xie X: Breast lesions: Quantitative diagnosis using ultrasound shear wave elastography - a systematic review and meta-analysis. Ultrasound Med Biol 42: 835-847, 2016.

21. Woo S, Suh CH, Kim SY, Cho JY and Kim SH: Shear-wave elastography for detection of prostate cancer: A systematic review and diagnostic meta-analysis. AJR Am J Roentgenol 209: 806-814, 2017.

22. Jiao Y, Dong F, Wang H, Zhang L, Xu J, Zheng J, Fan H, Gan H, Chen L and Li M: Shear wave elastography imaging for detecting malignant lesions of the liver: A systematic review and pooled meta-analysis. Med Ultrason 19: 16-22, 2017.

23. Gasparini G: Quantification of intratumoral vascularization predicts metastasis in human invasive solid tumors (Review). Oncol Rep 1: 7-12, 1994.

24. Zhang FJ and Han RL: The value of acoustic radiation force impulse (ARFI) in the differential diagnosis of thyroid nodules. Eur J Radiol 82: e686-e690, 2013.

25. Woo S, Kim SY, Cho JY and Kim SH: Shear wave elastography for detection of prostate cancer: A preliminary study. Korean J Radiol 15: 346-355, 2014.

26. Barr RG and Zhang Z: Shear-wave elastography of the breast: Value of a quality measure and comparison with strain elastography. Radiology 275: 45-53, 2015.

27. Pass B, Johnson M, Hensor EM, Gupta H and Robinson P: Sonoelastography of musculoskeletal soft tissue masses: A pilot study of quantitative evaluation. J Ultrasound Med 35: 2209-2216, 2016.

28. Pass B, Jafari M, Rowbotham E, Hensor EM, Gupta H and Robinson P: Do quantitative and qualitative shear wave elastography have a role in evaluating musculoskeletal soft tissue masses? Eur Radiol 27: 723-731, 2017.

29. Tavare AN, Alfuraih AM, Hensor EMA, Astrinakis E, Gupta H and Robinson P: Shear-wave elastography of benign versus malignant musculoskeletal soft-tissue masses: Comparison with conventional US and MRI. Radiology 290: 410-417, 2019.

30. Winn N, Baldwin J, Cassar-Pullicino V, Cool P, Ockendon M, Tins B and Jaremko JL: Characterization of soft tissue tumours with ultrasound, shear wave elastography and MRI. Skeletal Radiol 49: 869-881, 2020

31. Steffner RJ and Jang ES: Staging of bone and soft-tissue sarcomas. J Am Acad Orthop Surg 26: e269-e278, 2018. 